SLAC-PUB-8678

BABAR-PROC-00/21

October, 2000

\title{
B lifetime measurements with exclusively reconstructed B decays
}

\author{
Chih-hsiang Cheng \\ Department of Physics, Stanford University \\ Stanford, California 94305, USA \\ (for the BABAR Collaboration)
}

\begin{abstract}
Data collected with the BABAR detector at the PEP-II asymmetric B Factory at SLAC are used to measure the lifetimes of the $B^{0}$ and $B^{+}$mesons. The data sample consists of $8.3 \mathrm{fb}^{-1}$ collected near the $\Upsilon(4 \mathrm{~S})$ resonance. $B^{0}$ and $B^{+}$mesons are fully reconstructed in several exclusive hadronic decay modes to charm and charmonium final states. The $B$ lifetimes are determined from the flight length difference between the two $B$ mesons. The preliminary measurements of the lifetimes are

$$
\begin{aligned}
\tau_{B^{0}} & =1.506 \pm 0.052(\text { stat }) \pm 0.029 \text { (syst) ps } \\
\tau_{B^{+}} & =1.602 \pm 0.049(\text { stat }) \pm 0.035 \text { (syst) ps }
\end{aligned}
$$
\end{abstract}

and of their ratio is

$$
\tau_{B^{+}} / \tau_{B^{0}}=1.065 \pm 0.044 \text { (stat) } \pm 0.021 \text { (syst) }
$$

Contributed to the Proceedings of the DPF 2000 Meeting of the Division of Particles and Fields of the American Physical Society, 8/9/2000-8/12/2000, Columbus, Ohio 


\section{Introduction}

This paper presents preliminary measurements of the $B^{ \pm}$and $B^{0} / \bar{B}^{0}$ lifetimes and their ratio with data collected by the BABAR detector[1] at the PEP-II asymmetric-energy $B$ Factory. At the $\Upsilon(4 \mathrm{~S}), B$ mesons are produced in $B^{+} B^{-}$or $B^{0} \bar{B}^{0}$ pairs. In PEP-II, the center-of-mass frame is boosted roughly along the $z$ axis $(\beta \gamma \simeq 0.56)$. In this analysis, one of the $B$ mesons is fully reconstructed in a variety of clean hadronic decay modes to charm and charmonium final states. An inclusive technique is performed to reconstruct the decay vertex of the opposite $B$ meson, and the lifetime is determined from the distance along the $z$-axis between the decay vertices of the two $B$ mesons $\left(\Delta z=z_{\text {rec }}-z_{\mathrm{opp}}\right)$. The true value of $\Delta z$ is distributed exponentially with an average of $\langle|\Delta z|\rangle=(\beta \gamma)_{B}^{z} c \tau_{B} \simeq\left(p_{\Upsilon(4 \mathrm{~S})}^{z} / m_{\Upsilon(4 \mathrm{~S})}\right) c \tau_{B}$.

\section{Event sample and vertex reconstruction}

The data used in this analysis were collected by the BABAR detector at the PEP-II storage ring in the period from January to June 2000. The total luminosity is $7.4 \mathrm{fb}^{-1}$ collected at the $\Upsilon(4 \mathrm{~S})$ resonance and $0.9 \mathrm{fb}^{-1} 40 \mathrm{MeV}$ below the resonance. The number of produced $B \bar{B}$ pairs is estimated to be $8.4 \times 10^{6}$.

$B^{0}$ and $B^{+}$mesons are reconstructed in the following modes (and their charge conjugates): $B^{0} \rightarrow D^{(*)-} \pi^{+}, D^{(*)-} \rho^{+}, D^{(*)-} a_{1}^{+}, J / \psi K^{* 0}$ and $B^{+} \rightarrow \bar{D}^{(*) 0} \pi^{+}, J / \psi K^{+}, \psi(2 S) K^{+}$. All final state particles are reconstructed. The signal region for each decay mode in the selected sample is defined by the three standard deviation bands in the two-dimensional distribution of the kinematic variables, $\Delta E$ and $m_{\mathrm{ES}}{ }^{\text {}}$. The resolution on $m_{\mathrm{ES}}$ is about $3 \mathrm{MeV} / \mathrm{c}^{2}$, and that on $\Delta E$ varies from mode to mode between 12 and $40 \mathrm{MeV}$. The purities of final samples are approximately $90 \%$ (Fig.1).

A geometric and kinematic fit of the fully reconstructed $B_{\text {rec }}$ is performed and is required to converge. The vertex resolution ranges from 45 to $65 \mu \mathrm{m}$, depending on the mode. The vertex of the opposite $B$ is determined using all the tracks that are not associated with the $B_{\text {rec }}$, along with the $B_{\text {opp }}$ "pseudotrack", which is estimated from the momentum difference between $\Upsilon(4 \mathrm{~S})$ and $B_{\text {rec }}$, and the beamspot. The fitting procedure is repeated, after removing tracks and $\mathrm{V}^{0}$ decays that result in poor fits, until no track contributes more than a certain value to $\chi^{2}$. This algorithm gives one standard deviation errors of $115 \mu \mathrm{m}$ and biases around $25 \mu \mathrm{m}$ with Monte Carlo simulation. The Monte Carlo simulation is also used to study the $\Delta z$ resolution function of the residual $\delta(\Delta z)=(\Delta z)_{\text {measured }}-(\Delta z)_{\text {generated }}$ and the pull $\delta(\Delta z) / \sigma(\Delta z)$. Two-Gaussian fits successfully describe the Monte Carlo residual and pull distributions. They give a one standard deviation width of $130 \mu \mathrm{m}$ and a bias of $24 \mu \mathrm{m}$ for the residual. Because it is dominated by the $B_{\text {opp }}$ vertex, the resolution function shape is essentially the same for all modes we have considered.

\section{Lifetime fits and systematic uncertainties}

The lifetime is extracted from the $\Delta z$ distribution of the selected events with an unbinned maximum likelihood fit. The measurements performed on each event $i$ are represented by three input numbers:

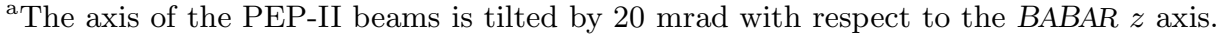

${ }^{\mathrm{b}} \Delta E=E_{r e c}^{*}-E_{b}^{*}, m_{\mathrm{ES}}=\sqrt{E_{b}^{* 2}-\boldsymbol{p}_{\mathrm{rec}}^{* 2}}$ where $E_{r e c}^{*}$ and $\boldsymbol{p}_{\mathrm{rec}}^{*}$ are the measured $B$ candidate energy and momentum, and $E_{b}^{*}$ is the beam energy, all defined in the center-of-mass frame.
} 
$(\Delta z)_{i}$, its error $\sigma_{i}$, and the probability for an event $i$ to come from signal based on the $m_{\mathrm{ES}}$ spectrum. All events that satisfy $5.2<m_{\mathrm{ES}}<5.3 \mathrm{GeV} / c^{2}$ and have $\Delta E$ in the signal region are input to the fit. The fit determines the $B$ lifetime, the proportion of outliers, and two sets of parameters that describe the resolution function and the $\Delta z$ shape of the background. The signal distribution is represented by a convolution of the theoretical $\Delta z$ distribution (two exponential wings) and the pull representation of the $\Delta z$ resolution function. A function of the form $G \otimes(1+E)$ is used?. The background $\Delta z$ distribution is described by the sum of a single Gaussian and two independent exponential tails, one for positive $\Delta z$ and one for negative $\Delta z$. The outliers are described by a wide symmetric Gaussian with a fixed width of $2500 \mu \mathrm{m}$.

We fit the $\Delta z$ distributions of $B^{0}$ and $B^{+}$samples simultaneously with a single set of parameters for the resolution function but different sets of parameters (one per charge) to describe the lifetime, the background and the outliers (Fig.1). The lifetime ratio is determined with a similar combined fit with the $B^{0}$ lifetime and the ratio being free parameters. The statistical errors are about $3.5 \%$. About $2 \%$ of the statistical error is due to the high correlation between lifetime and the $\Delta z$ resolution function parameters.

The dominant systematic uncertainties are the limited Monte Carlo statistics for checking $\Delta z$ distortion due to event selection, the modeling of $\Delta z$ outliers, the length scale measured along $z$, and the background modeling. Each contributes about $1 \%$ to the systematic uncertainty. All other systematic uncertainties we have studied, including the choice of parameterization for the resolution function and errors on the boost measurements, are less than $0.5 \%$. The approximation of using the $\Upsilon(4 \mathrm{~S})$ boost for both $B$ mesons in an event results in a $0.4 \%$ shift. The central values of the lifetimes are corrected for this shift.
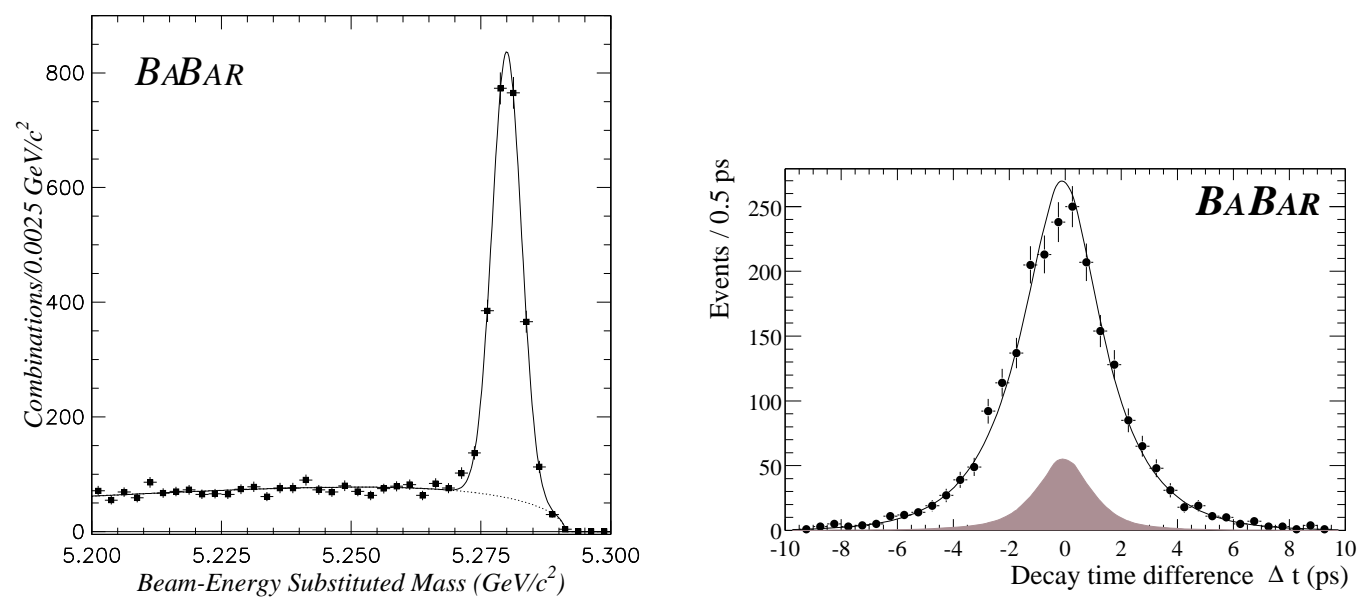

Figure 1: Left: $m_{\mathrm{ES}}$ distribution for all the hadronic modes for $B^{0}$, fit to the sum of a Gaussian distribution and the ARGUS background parameterization[2]. The total yields in all $B^{0}$ and $B^{+}$ modes are $2210 \pm 58$ and $2261 \pm 53$ (not shown), respectively. Right: $\Delta t$ distribution for $B^{0} / \bar{B}^{0}$. The result of the lifetime fit is superimposed. The background is shown by the hatched distribution.

${ }^{\mathrm{c}}$ The sum of an unbiased Gaussian and the convolution of the same Gaussian with a decaying exponential. This resolution function leads to the smallest overall error with present statistics. 
The preliminary results for the $B$ meson lifetimes are

$$
\tau_{B^{0}}=1.506 \pm 0.052 \pm 0.029 \mathrm{ps}, \quad \tau_{B^{+}}=1.602 \pm 0.049 \pm 0.035 \mathrm{ps}
$$

and for their ratio is

$$
\tau_{B^{+}} / \tau_{B^{0}}=1.065 \pm 0.044 \pm 0.021
$$

The first error is statistical and the second is systematic. The results are consistent with previous $B$ lifetime measurements[3] and competitive with the most precise ones.

\section{References}

[1] Technical Design Report, BABAR Collaboration (1995).

[2] ARGUS Collaboration, H. Albrecht et al., Z. Phys. C 48, 543 (1990).

[3] Particle Data Group, D. E. Groom et al., Eur. Phys. Jour. C 15, 1 (2000). 